\title{
Differential diagnoses of COVID-19 pneumonia: the current challenge for the radiologist - a pictorial essay
}

\author{
Alessia Guarnera ${ }^{1 *+} \mathbb{0}$, Pierfrancesco Podda ${ }^{2 \dagger}$, Elena Santini ${ }^{2}$, Pasquale Paolantonio ${ }^{2}$ and Andrea Laghi ${ }^{1}$
}

\begin{abstract}
Background: COVID-19 pneumonia represents the most severe pandemic of the twenty-first century and has crucial clinical, social and economical implications. The scientific community has focused attention and resources on clinical and radiological features of COVID-19 pneumonia. Few papers analysing the vast spectrum of differential diagnoses have been published.

Main body: Complexity of differential diagnosis lays in the evidence of similar radiological findings as ground-glass opacities, crazy paving pattern and consolidations in COVID-19 pneumonia and a multitude of other lung diseases. Differential diagnosis is and will be extremely important during and after the pandemic peak, when there are fewer COVID-19 pneumonia cases. The aim of our pictorial essay is to schematically present COVID-19 pneumonia most frequent differential diagnoses to help the radiologist face the current COVID-19 pneumonia challenge.

Conclusions: Clinical data, laboratory tests and imaging are pillars of a trident, which allows to reach a correct diagnosis in order to grant an excellent allocation of human and economical resources. The radiologist has a pivotal role in the early diagnosis of COVID-19 pneumonia because he may raise suspicion of the pathology and help to avoid COVID-19 virus spread.
\end{abstract}

Keywords: COVID-19, Coronavirus, Pneumonia, Differential diagnosis, Computed tomography

\section{Key points}

1. COVID-19 pneumonia generally passes through four stages characterised by different radiological features.

2. Ground-glass opacities, crazy paving, consolidations and fibrotic striae are common to various pathologies.

3. Radiological differential diagnosis roots in the localisation of these radiological features in the lung and in their timing of occurrence.

\footnotetext{
*Correspondence: guarneraalessia@gmail.com

${ }^{\dagger}$ Alessia Guarnera and Pierfrancesco Podda have contributed equally to this work

1 Department of Surgical and Medical Sciences and Translational Medicine, Sapienza University of Rome - Sant'Andrea University Hospital, Via di Grottarossa, 1035-1039, 00189 Rome, Italy

Full list of author information is available at the end of the article
}

4. Presence of pulmonary nodules, pleural effusions and adenopathies is suggestive of alternative diagnoses.

\section{Background}

Since December 2019, when a novel coronavirus has been identified in Wuhan [1] and then termed COVID19 [2], the viral pneumonia has been spreading around the globe, and on March 11, 2020, the WHO defined COVID-19 pneumonia as pandemic [3].

Since COVID-19 outbreak, the scientific community has focused on clinical and radiological features of COVID-19 pneumonia, while few papers have analysed the vast spectrum of COVID-19 pneumonia differential diagnoses [4-6].

Complexity of differential diagnosis is high, especially in populations not drastically affected by COVID-19, and 
will be higher after the peak of pandemic, when there are few cases.

The crucial and challenging role of the radiologist is and will be to raise suspicion of COVID-19 pneumonia, to guarantee a correct allocation of human and economic resources, and to prevent COVID-19 virus spread.

The aim of our pictorial essay is to schematically present COVID-19 pneumonia most frequent differential diagnoses to help the radiologist face the current COVID-19 pneumonia challenge.

\section{Main text}

\section{COVID-19 pneumonia}

COVID-19 pneumonia is a virulent pulmonary pathology, with high person-to-person transmission [7] through the inhalation of virus which infects alveolar and endothelium cells by linking to the receptor for ACE II [8].

Chest radiography has been defined as insensitive in mild or early COVID-19 infection [9, 10]; therefore, it does not represent an efficient radiological tool to reach an early and correct diagnosis. Its main role, especially as portable chest radiography and in relation to COVID19 pneumonia, is pneumonia monitoring after diagnosis or alternative diagnosis assessment $[9,11]$, because it is time-effective and cost-effective and the equipment decontamination is easier and quicker compared to CT scan. These characteristics make chest radiography a useful technique to minimise the risk of cross-infection and to avoid radiological service availability disruption, which happens during CT scan decontamination [9].

CT is more sensitive for early COVID-19 pneumonia detection, disease progression monitoring and alternative diagnosis assessment [9]. In the setting of COVID-19 pandemic, the greater sensitivity of $\mathrm{CT}$ is pivotal to guarantee an early diagnosis and isolation of infected patients [9].

Feng Pan identified four stages of lung involvement on chest CT, even if semiological findings may coexist in phase transitions [12] (Table 1):

- Early phase (0-4 days) or stage 1 : ground-glass opacities [12] (Fig. 1a);

- Progressive phase (5-8 days) or stage 2: crazy paving pattern $[12,13]$, extensive ground-glass opacities and small consolidations [12] (Fig. 1b-d);

- Peak phase (9-13 days) or stage 3 [12]: consolidative foci, sometimes surrounded by an halo of groundglass (halo sign) [12] (Fig. 1e). Atoll sign or reversed halo sign [14] has been described [13];

- Absorption phase or stage 4 ( $\geq 14$ days) [12]: groundglass opacities and linear consolidation that may be interpreted as a process of repair and reorganisation, partially mediated by an organisational pneumonia, which is a stereotypical response to lung injury [4] (Fig. 1f).

Further characteristic and mostly constant findings in COVID-19 pneumonia are [12, 14, 15] (Table 1):

- subpleural, bilateral or less frequently unilateral, opacities, which are commonly located in the inferior lobes;

- possible evidence of peripheral pulmonary vessel widening;

- rarity of pleural effusions, pulmonary nodules and mediastinal lymphadenopathies.

Acute respiratory distress syndrome (ARDS) represents a dire complication of severe cases of COVID19 pneumonia $[12,16]$ and has been speculated to be secondary to pulmonary microvascular obstructive thrombo-inflammatory syndrome [17]. Activation of the coagulation system has been shown to be relevant in the pathogenesis of ARDS, and therefore, therapy with heparin has been suggested as treatment for severe forms of COVID-19 pneumonia [18] because it decreases mortality [19].

Radiologically ARDS presents an anteroposterior gradient and dependent consolidations coexisting with widespread ground-glass attenuation [20] (Fig. 1g).

\section{Differential diagnoses}

There is a wide spectrum of possible differential diagnoses for COVID-19 pneumonia, and it is always necessary to consider a triptych of clinical, laboratory and radiological data to reach the correct diagnosis (Table 2).

\section{Infective pneumonia}

Respiratory infections are the most common illnesses occurring in humans [21], the most common being community-acquired pneumonia.

\section{Bacterial pneumonia}

Radiological features for differential diagnosis:

- single consolidation with air bronchogram, usually presenting as lobar pneumonia and not exceeding pleural cleavages (typical pneumonia: Staphylococcus aureus, Streptococcus pneumoniae, Moraxella catarrhalis, Enterobacteriaceae) [5, 21, 22] (Fig. 2a);

- multifocal pneumonia presents with ground-glass opacities or consolidations and usually coexists with centrilobular nodules and thickening of bronchovascular bundles (atypical pneumonia: Mycoplasma pneumoniae, Chlamydia pneumoniae) [30] (Fig. 2b); 


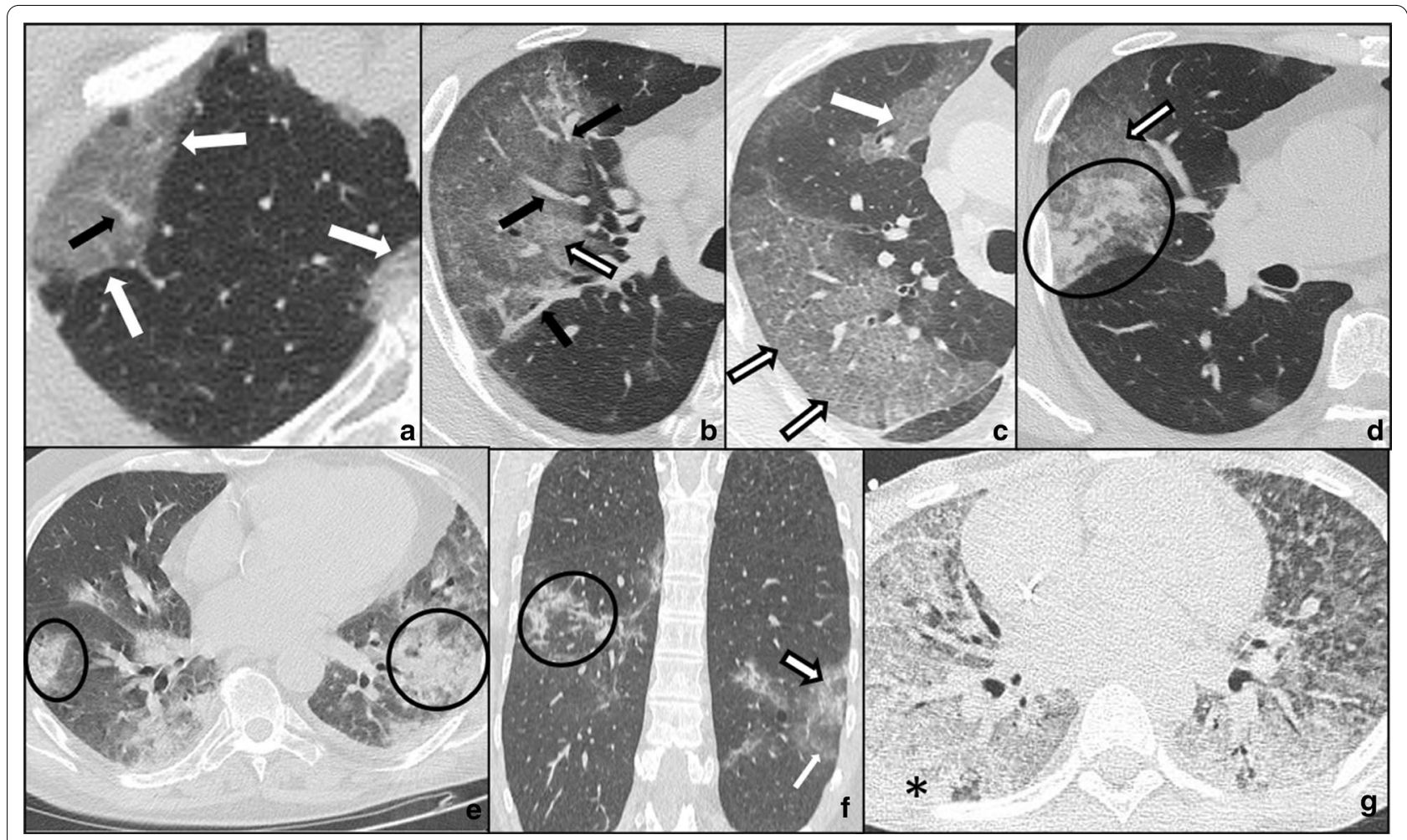

Fig. 1 a-g COVID-19 pneumonia phases. HRTCs of patients showing the different phases and possible evolutions of COVID-19 pneumonia. In a, two subpleural, sharply demarked ground-glass opacities (white arrows in a) indicate phase 1. There is evidence of concomitant widening of peripheral vessels (black arrow in $\mathbf{a}$ ). In $\mathbf{b}$ and c, subpleural extensive areas of ground glass (white arrow in $\mathbf{c}$ ) and crazy paving pattern (black bordered white arrows in $\mathbf{b}, \mathbf{c}$ ) are referable to phase 2. Black arrows in $\mathbf{b}$ indicate dilatated segmental and subsegmental vessels. Phase 2 may also be characterised by extensive areas of crazy paving (black bordered white arrow in $\mathbf{d}$ ), in which small consolidative foci are evident (black circle in d); In e, dependent and non-dependent consolidations (black circles in e) are hallmarks of phase 3. Phase 4 (f) presents as irregular consolidative foci (black circle in $\mathbf{f}$ ) coexisting with confluent ground-glass opacities (white arrow in $\mathbf{f}$ ) and crazy paving pattern (black bordered white arrow in $\mathbf{f}$ ), whereas ARDS, which represents a possible complication COVID-19 pneumonia, is characterised by dependent consolidations (black asterisk in $\mathbf{g}$ ) and widespread ground-glass attenuation

Table 1 Radiological findings in COVID-19 pneumonia

\begin{tabular}{|c|c|c|c|c|c|}
\hline Stage & Phase & Timing (days) & Predominant radiological findings & Additional findings & $\begin{array}{l}\text { Spatial distribution of radiological } \\
\text { findings }\end{array}$ \\
\hline 1 & Early & $0-4$ & Ground-glass opacities & \multirow{3}{*}{$\begin{array}{l}\text { Peripheral vessel widening } \\
\text { Halo sign } \\
\text { Atoll sign or reversed halo sign } \\
\text { Overlapping of radiological findings } \\
\text { in different phases }\end{array}$} & \multirow{4}{*}{$\begin{array}{l}\text { Bilateral } \\
\text { Peripheral/subpleural } \\
\text { Centroparenchymal (atypical) } \\
\text { Lower lobes prevalence }\end{array}$} \\
\hline 2 & Progressive & $5-8$ & $\begin{array}{l}\text { Crazy paving pattern, ground-glass } \\
\text { opacities and small consolidations }\end{array}$ & & \\
\hline 3 & Peak & $9-13$ & Consolidative foci & & \\
\hline 4 & Absorption & $\geq 14$ & $\begin{array}{l}\text { Ground-glass opacities and linear } \\
\text { consolidation }\end{array}$ & $\begin{array}{l}\text { Rarity of: lymphadenopathies, pleu- } \\
\text { ric effusions, pulmonary nodules }\end{array}$ & \\
\hline
\end{tabular}

COVID-19 pneumonia has been divided into four stages, according to Feng Pan [12], depending on the timing of typical radiological findings occurrence. Spatial distribution of radiological findings and additional findings is of primary importance to reach a correct diagnosis [12, 14, 15]

- Mycoplasma pneumoniae may cause multiple, bilateral ground-glass opacities as COVID-19, but mainly occurs in children [5], while COVID-19 pneumonia is less common and severe in the paediatric population [23].
- additional findings as centrilobular nodules, cavitations and pneumatoceles (more common in S. aureus pneumonia) [22];

- hilomediastinal lymphadenomegalies [5, 21, 22];

- pleural effusions [5, 21, 22]. 


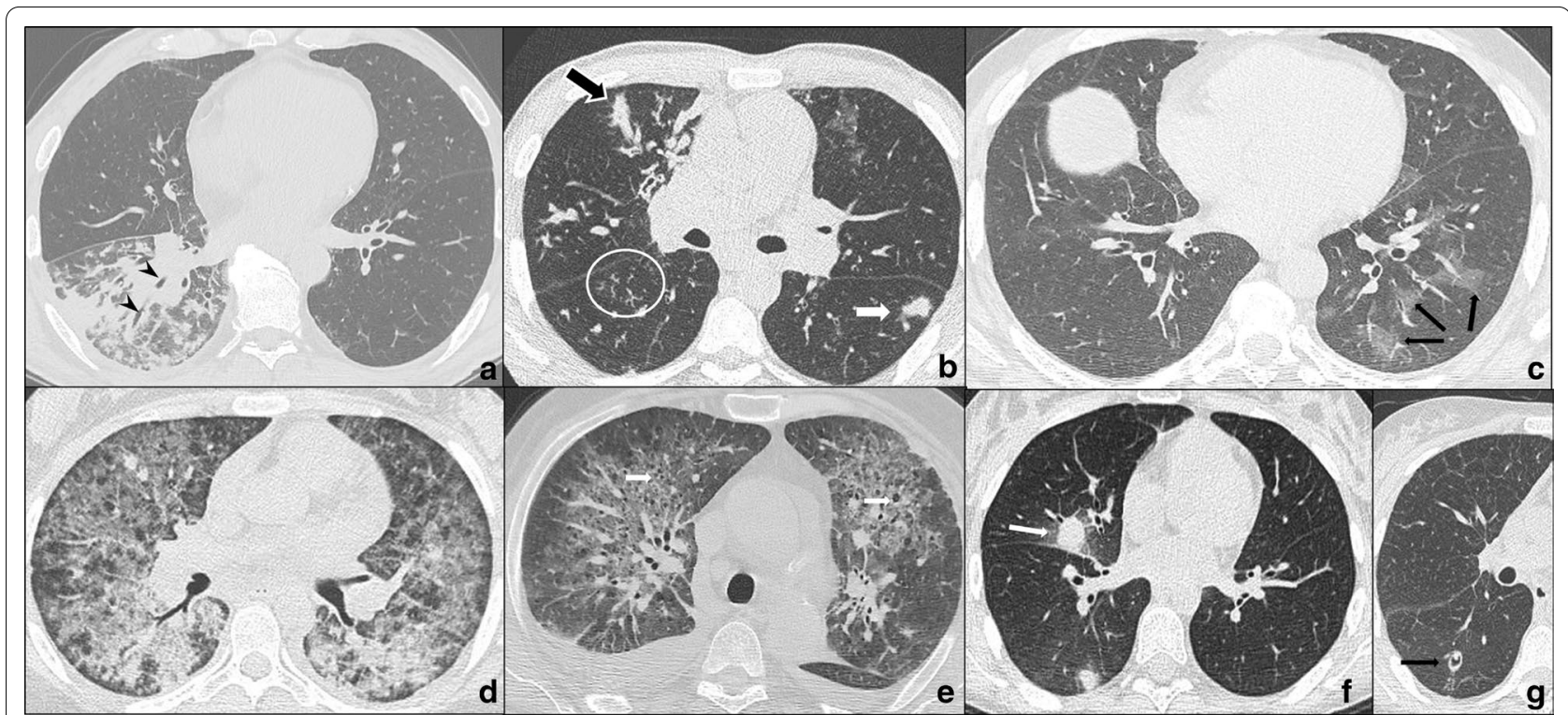

Fig. 2 a-g COVID-19 pneumonia differential diagnosis: infective pneumonia. HRTCs of infective pneumonias. In a, S. Pneumoniae lobar pneumonia shows consolidations with air bronchogram (black arrowheads in $\mathbf{a}$ ), while in $\mathbf{b}$, atypical pneumonia presents as multiple nodular (white arrow in b), tubular (white bordered black arrow in b) consolidative foci and centrilobular nodules (white circle in b). In c, HRTC showing centroparenchymal ground-glass opacities (black arrows in c) in patient affected by Influenza type A pneumonia. Patients affected by HIV and pneumocistis jiroveci pneumonia in $\mathbf{d}$ and $\mathbf{e}$, and presenting with bilateral, widespread crazy paving and ground-glass opacities on HRTC during acute pneumonia and perihilar pneumatoceles (white arrows in $\mathbf{e}$ ) with pleural effusions in a long-lasting pneumonia. HRCTs of patient affected by angioinvasive aspergillosis at baseline (f) and after 7 days ( $(\mathbf{g})$. At baseline, two consolidations rimmed by ground-glass halo are evident in $\mathbf{f}$ (white arrow in $\mathbf{f}$, halo sign), while after therapy, excavated nodules showing "air crescent sign" (black arrow in $\mathbf{g}$ ) indicate the remission phase

\section{Viral pneumonia}

Viral pneumonia represents a various entity, and it is mainly the current epidemic context which suggests COVID-19 origin [4]. Treatment has been proved to be similar, currently [5].

Radiological features for differential diagnosis:

- preferential centro-parenchymal involvement (Influence type A, Adenovirus, Hantavirus) [21] (Fig. 2c);

- additional findings as centrilobular nodules and bronchial wall thickening (RSV, MERS, Influence type A) [21];

- coexisting pulmonary oedema (Hantavirus) [24];

- pleural effusions (RSV, MERS) [21];

- hilomediastinal lymphadenomegalies (Influence type A) [21].

\section{Pneumocystis jiroveci pneumonia}

It is an opportunistic fungal pneumonia, mostly affecting immunodeficient patients affected by AIDS or undergoing immunosuppressive therapies (Fig. 2d, e).

Anamnesis and laboratory tests are of use, but often not sufficient for a differential diagnosis with COVID19 pneumonia. Radiological features for differential diagnosis:
- symmetrical, centroparenchymal and perihilar, confluent ground-glass opacities, generally with subpleural sparing [25] and a predilection for the upper lobes [26];

- rarity and late onset of crazy paving pattern;

- additional findings as nodules and pneumatoceles.

\section{Angioinvasive aspergillosis}

Angioinvasive aspergillosis is caused by Aspergillus Fumigatus [27] and generally affects immunocompromised patients with severe neutropenia [28].

Radiological features for differential diagnosis:

- ground-glass opacities and crazy paving pattern are not typical and do not precede consolidations, which frequently present a surrounding ground-glass halo (halo sign) [29] (Fig. 2f);

- in case of consolidations with no ground-glass halo and absence of other ground-glass opacities, COVID-19 pneumonia is unlikely;

- presence of air crescent sign [13, 27, 29] (Fig. 2g);

- lymphadenomegalies and pleural effusions [29]. 


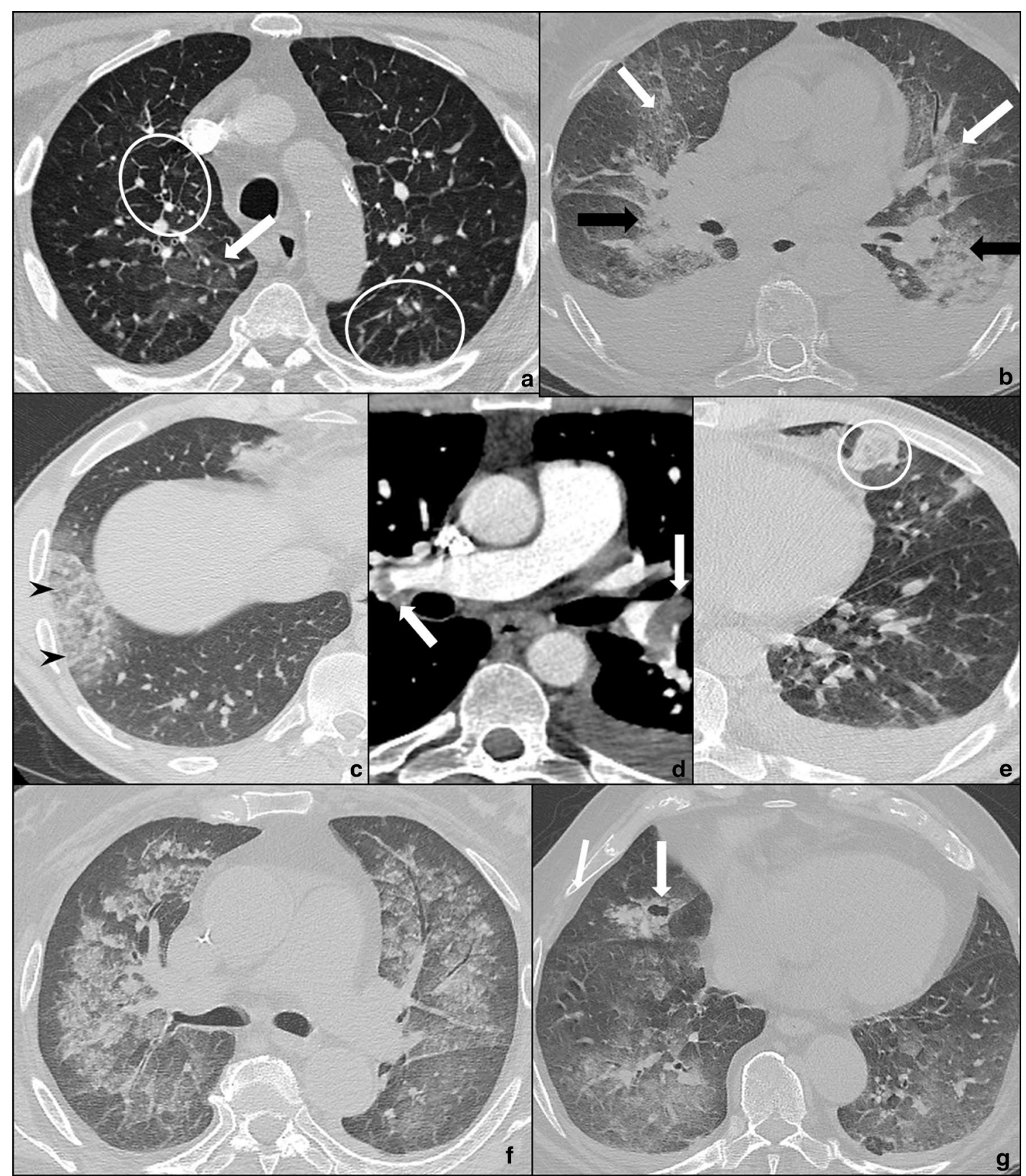

Fig. 3 a-g COVID-19 pneumonia differential diagnosis: cardiovascular pathologies. HRCTs of patients presenting with interstitial oedema (a) and interstitial-alveolar oedema (b). In $\mathbf{a}$, there is extensive smooth thickening of interlobular septa (white circles in $\mathbf{a}$ ) coexisting with ground-glass opacities (white arrow in $\mathbf{a}$ ); in b, confluent perihilar consolidations (black arrows in $\mathbf{b}$ ) coexist with crazy paving pattern (white arrows in b) and bilateral pleural effusions. HRTC (c, e) and AngioCT (d) in patients affected by APE: bilateral emboli (white arrows in d) caused two pulmonary infarcts manifesting, respectively, as an area of crazy paving (black arrowheads in c) and a consolidation with a hypodense core ("reversed halo sign", white circle in e). HRTC of a patient affected by Granulomatosis with polyangiitis (f, $\mathbf{g})$ showing massive centroparenchymal DAH (f) and an excavated nodule (white arrow in $\mathbf{g}$ ) 


\section{Cardiovascular pathologies Pulmonary oedema}

Two pathophysiologic and radiologic phases are recognised in cardiogenic pulmonary oedema development [30]: interstitial (Fig. 3a) and alveolar (Fig. 3b).

Apart from anamnesis, radiological features for differential diagnosis are [4, 30]:

- possible coexistence of ground-glass opacities, crazy paving pattern and consolidations with different timing of occurrence respect to COVID-19 pneumonia, crazy paving being the first pattern to be appreciated in interstitial oedema;

- diffuse, bilateral, centro-parenchymal crazy paving and ground-glass opacities with subpleural preservation. Consolidations are late findings and generally coexist with pleural effusions;

- bilateral pleural effusions, more evident in the alveolar phase of oedema;

- mediastinal lymphadenomegalies;

- cardiomegaly.

Chen et al. [31] reported acute myocarditis related to COVID-19 infection. The overlapping of findings between COVID-19 pneumonia and pulmonary oedema should always raise suspicion of myocarditis, especially in young patients [4].

\section{Acute pulmonary embolism (APE)}

APE, which is is commonly secondary to a phlebothrombosis, most frequently of the lower limbs, has an acute clinical onset [32] and may cause pulmonary infarctions (Fig. 3c, e).

It is crucial to collect a proper anamnesis and perform radiological examinations as AngioCT to identify luminal defects of pulmonary vessels (Fig. 3d). Radiological features for differential diagnosis are [32]:

- different phases of infarction maturation, in correlation to the onset time;

- segmental shape of infarctions are located in the vascular territories of the occluded vessels;

- presence of vessel embolus, vessel occlusion and residual peripheral clot deposition.

It has been speculated on extensive pulmonary thromboembolism in severe cases of COVID-19 pneumonia [17, 32, 33], mediated by COVID-19 endothelial tropism [8]. Luckily, heparin, which is the first therapy for APE, has shown to have effect on patients affected by severe forms of COVID-19 pneumonia [18, 19].

COVID-19 pneumonia and pulmonary embolism may coexist; in particular, a person might present with the symptoms of acute pulmonary embolism and actually suffer of fulminant pulmonary embolisation but be also infected with COVID-19 being asymptomatic from the infection [34]. GGO with a typical appearance on CT might be the only sign in this person [34].

\section{Vasculitis}

Vasculitis is an inflammatory process in which immune effector cells infiltrate blood vessels and surrounding

Table 2 Radiological features of pathologies in differential diagnosis with COVID-19 pneumonia

\begin{tabular}{|c|c|c|c|c|}
\hline Pathologies & & Ground glass & Crazy paving & Consolidations \\
\hline \multirow[t]{4}{*}{ Infective pneumonia } & Bacterial & R (ATYPICAL) & OD/A & C (TYPICAL) \\
\hline & Viral & C & $\mathrm{OD} / \mathrm{A}$ & $\mathrm{R}$ \\
\hline & Fungal: pneumocystis jiroveci pneumonia & C & $\mathrm{R}$ & $\mathrm{R}$ \\
\hline & Fungal: angioinvasive aspergillosis & $(\mathrm{HALO})$ & $\mathrm{OD} / \mathrm{A}$ & C \\
\hline \multirow[t]{3}{*}{ Cardiovascular } & Acute pulmonary oedema & C (INTERSTITIAL) & C (INTERSTITIAL) & C (ALVEOLAR) \\
\hline & Acute pulmonary embolism and infarctions & C & C & C \\
\hline & Vasculitis & C (HAEMORRAGE) & C (REABSORPTION) & C (MASSIVE HAEMORRAGE) \\
\hline Hypersensitivity pneumonia & & C & OD/A & $\mathrm{R}$ \\
\hline \multirow[t]{3}{*}{ Eosinophilic pneumonia } & Simple pulmonary eosinophilia & C & OD/A & C \\
\hline & Acute eosinophilic pneumonia & C & C & $\mathrm{R}$ \\
\hline & Chronic eosinophilic pneumonia & $R$ & $\mathrm{R}$ & C \\
\hline \multirow[t]{2}{*}{ Aspiration pneumonia } & Fluid-related ab ingestis pneumonia & C & OD/A & C \\
\hline & Chronic lipoid pneumonia & C & C & C \\
\hline Alveolar proteinosis & & OD/A & C & $R$ \\
\hline
\end{tabular}

Analysed pathologies usually share at least one radiological feature, among ground-glass areas, crazy paving opacities and consolidations, with COVID-19 pneumonia. These findings may be either common or rare presentation of pathologies or be occasionally described/absent. The timing of these features presentation frequently varies with respect to COVID-19 pneumonia characteristic phases

C, common; R, rare; OD/A, occasionally described/absent 
tissues [35, 36]. ANCA-associated small vessel vasculitis frequently present with predominant pulmonary involvement and may cause diffuse alveolar haemorrhage (DAH) [13, 37] (Fig. 3f, g).

Anamnesis and laboratory tests may be useful to prove a clinical suspect. Radiological features for differential diagnosis are:

- DAH, presenting as ground-glass opacities or consolidative foci if bleeding is massive, is more prominent in the perihilar region and in the inferior lobes [13, 37];

- DAH presents different timing of presentation, crazy paving being the last pattern to be appreciated [37, 38];

- symmetric, bilateral ground-glass opacities or consolidations, which tend to be migratory or transient (EGPA: eosinophilic granulomatosis with polyangiitis) [13, 37];

- typical vasculitis features, as pulmonary artery aneurysms and excavated nodules may be present and are more common in granulomatosis with polyangiitis, but rarely present in EGPA [13, 37];
- coexistence of bronchial and tracheal thickening in granulomatosis with polyangiitis;

- coexistence of bronchial and bronchiolar thickening or centrilobular nodules in EGPA [37, 38];

- pulmonary oedema findings, secondary to cardiac involvement in EGPA [10, 26];

- pleural effusions $[13,37]$.

\section{Hypersensitivity pneumonia}

It is an interstitial pathology caused by repetitive inhalation of and sensitization to a wide range of inorganic and organic antigens [39] in relation to occupational or environmental reasons. Usually it is divided into acute, subacute and chronic forms.

Radiological features for differential diagnosis (Fig. 4a):

- centroparenchymal and centrilobular ground-glass opacities [40];

- rarity of crazy paving pattern and consolidations [41, 42];

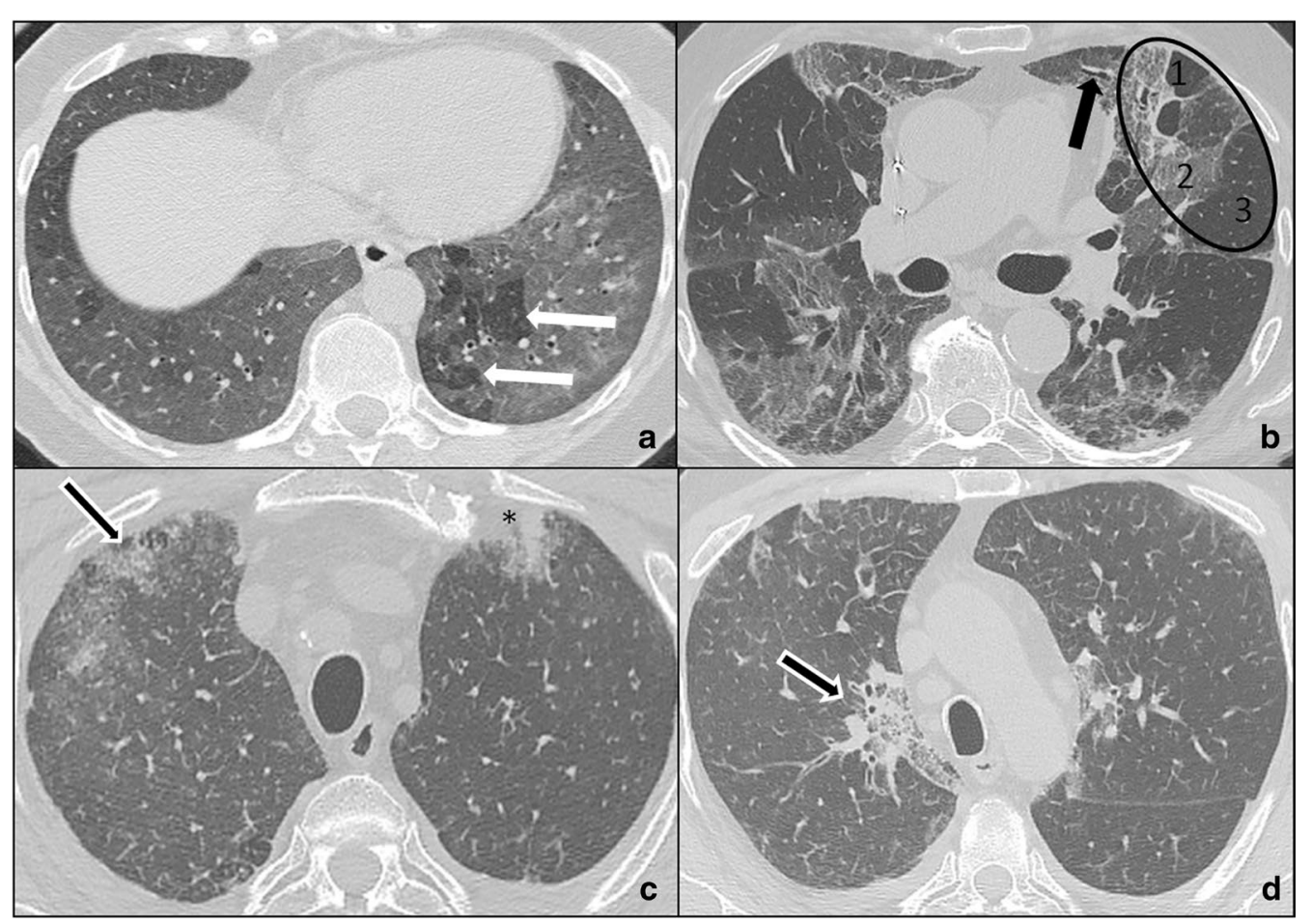

Fig. 4 a-d COVID-19 pneumonia differential diagnoses: hypersensitivity pneumonia and eosinophilic pneumonia. HRTCs of a patient affected by hypersensitivity pneumonia at baseline (a) and after two years (b): acute/subacute hypersensitivity pneumonia is characterised by diffuse ground-glass opacities clearly demarcated from air trapping areas (white arrows in $\mathbf{a}$ ); chronic hypersensitivity pneumonia presents with architectural distortions and traction bronchiectases (black arrow in $\mathbf{b})$. The triad of mosaic oligemia ( 1 in $\mathbf{b}$ ), ground-glass opacities ( 2 in $\mathbf{b}$ ) and normal pulmonary parenchyma ( 3 in $\mathbf{b}$ ) is defined as "headcheese sign" (black oval in $\mathbf{b}$ ). HRTC of acute eosinophilic pneumonia (c, d) showing confluent crazy paving opacities (white bordered black arrows in c, d) coexisting with consolidations (black asterisk in c) and slight bilateral pleural effusions 
- coexistence of other radiological findings as mosaic oligemia, cysts, centrilobular emphysema and centrilobular micronodules [40, 43];

- "headcheese sign," which represents the coexistence of patchy ground-glass opacities, preserved pulmonary regions, and air trapping on HRCT [44] (Fig. 4b);

- centrilobular fibrosis, architectural distorsions, traction bronchiectases and honeycombing in the chronic stage [41, 45];

- mediastinal lymphadenomegalies.

\section{Eosinophilic pneumonia}

Eosinophilic pneumonia represents a various group of pulmonary disorders associated with peripheral or tissue eosinophilia. Laboratory tests and anamnesis are crucial for a correct differential diagnosis [46-48]. HRTC is important to raise suspicion and avoid misdiagnoses.

Radiological features for differential diagnosis are (Fig. 4c, d):

- migrant lesions with absence of crazy paving pattern in SPE (simple pulmonary eosinophilia, also known as Loeffler syndrome) [46];

- pleural effusions, centrilobular nodules and thickening of bronchovascular bundles in AEP (acute eosinophilic pneumonia) $[46,49,50]$;

- centrilobular consolidations with rarity of groundglass opacities and crazy paving pattern in CEP (chronic eosinophilic pneumonia) [46, 51, 52];

- presence of additional findings in CEP as nodules, atelectasis, band-like opacities and pleural effusions $[46,51]$.

\section{Aspiration pneumonia}

It is caused by the aspiration of different substances into the airways and lungs [53]. Radiological findings may vary, but frequently anamnesis is sufficient for the diagnosis.

To the purpose of our paper, we will focus on fluid aspiration pneumonia and on lipoid pneumonia.

\section{Fluid aspiration pneumonia}

Frequently, these patients are dysfagic and their meals are liquid. Radiological features for differential diagnosis are [54] (Fig. 5a):

- dependent ground-glass opacities;

- in the late phases, with recurrence of aspiration, fibrotic architectural distortions.

\section{Lipoid pneumonia}

It is an acute or chronic, reactive pneumonia resulting from endogenous lipid accumulation or from exogenous lipid aspiration [55, 56]. Chronic lipoid pneumonia requires differential diagnosis with COVID-19 pneumonia, while anamnesis is generally sufficient for a clear diagnosis of acute pneumonia.

Anamnesis of lipid inhalation or fat storage disease is necessary to diagnose a lipoid pneumonia. Comparison with previous chest exams is suggested.

Radiological features for differential diagnosis are (Fig. 5b):

- ground-glass opacities and consolidations predominantly involve the middle and inferior lobes [54];

- consolidations typically present very low CT attenuation in relation to their fat content [57] (Fig. 5c);

- fibrosis during chronic stage $[55,58]$.

\section{Pulmonary alveolar proteinosis (PAP)}

It is a syndrome caused by progressive accumulation of surfactant in pulmonary alveoli and may be primary in most cases or secondary to toxic inhalation syndromes, haematologic neoplasms and immune deficiency [59, 60]. Anamnesis, laboratory tests and comparison with previous HRCTs are helpful.

Radiological features for differential diagnosis are (Fig. 5d, e):

- mainly centro-parenchymal and perihilar crazy paving areas [61];

- juxtaposition of severely affected secondary lobules and normal secondary lobules;

- rarely, consolidations with air bronchogram in severe forms [61];

- fibrotic alterations in advanced stage;

- pleural effusions, cardiomegaly and lymphadenomegalies, which are features of complicated PAP.

\section{Drug-induced lung disease}

Anamnesis of drug taking or recreative drug abuse is crucial for diagnosis and institution of appropriate treatment [62].

Among the most frequent causative drugs and the related most common pulmonary diseases we cite [62, 63]: amiodarone and methotrexate (organising pneumonia) [64]; immunosuppressants as sirolimus and everolimus (hypersensitivity pneumonia) [65]; heroin (eosinophilic pneumonia, pulmonary haemorrhage, 


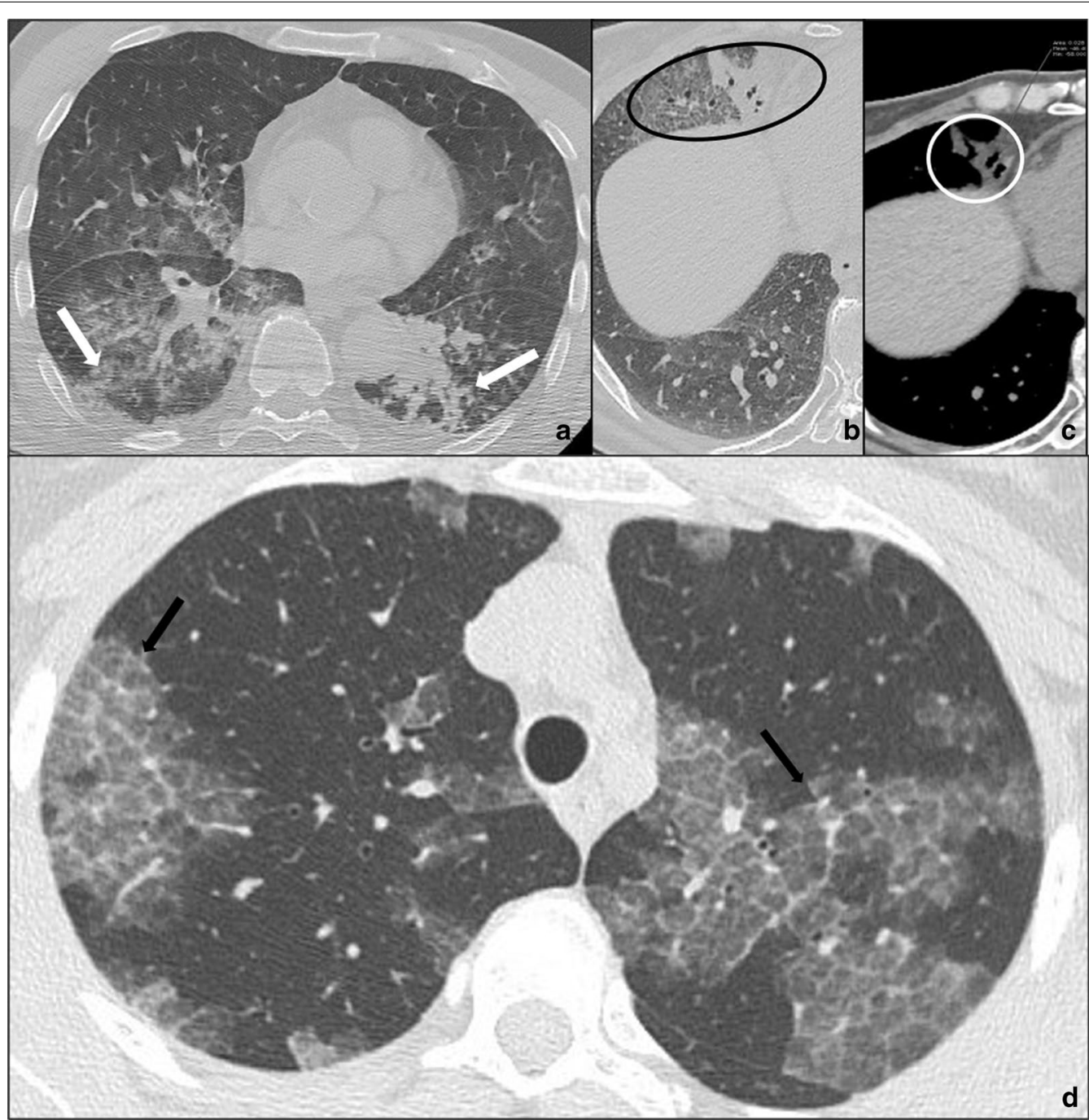

Fig. 5 a-d COVID-19 pneumonia differential diagnoses: aspiration pneumonia, pulmonary alveolar proteinosis. HRTCs of two patients affected by fluid aspiration pneumonia presenting with bilateral, dependent and confluent consolidative foci (white arrows in a) and chronic lipoid pneumonia (b, c), showing a low density consolidation (mean:- $46 \mathrm{UH}$ in white circle in c) surrounded by a crazy paving halo (black oval b). Pulmonary alveolar proteinosis is characterised by bilateral areas of crazy paving (black arrows in $\mathbf{d}$ ) and a peculiar juxtaposition of severely affected secondary lobules and normal secondary lobules

pulmonary oedema) [66-68]; cocaine (pulmonary oedema) [69].

Radiological features for differential diagnosis of cited pathologies have already been analysed in the previous chapters.

\section{Conclusions}

COVID-19 pneumonia early diagnosis is crucial to avoid the spread of the virus in a disease that may range from asymptomatic to extremely severe and to favour an optimal allocation of human and economic resources. In this context, the role of the radiologist is pivotal and challenging, because he filters patients and identifies possible cases of COVID-19 pneumonia.

\section{Abbreviations}

AEP: Acute eosinophilic pneumonia; APE: Acute pulmonary thromboembolism; ARDS: Acute respiratory distress syndrome; CEP: Chronic eosinophilic pneumonia; DAH: Diffuse alveolar haemorrhage; EGPA: Eosinophilic granuIomatosis with polyangiitis; PAP: Pulmonary alveolar proteinosis; SPE: Simple pulmonary eosinophilia.

\section{Authors' contributions}

AG has made substantial contributions to the conception and design of the work; the acquisition, analysis and interpretation of data; and the realisation of the manuscript. AG has approved the submitted version (and any substantially modified version that involves the author's contribution to the study) and has agreed both to be personally accountable for the author's own contributions 
and to ensure that questions related to the accuracy or integrity of any part of the work, even ones in which the author was not personally involved, are appropriately investigated, resolved, and the resolution documented in the literature. PP has made substantial contributions to: the conception and design of the work; the acquisition, analysis and interpretation of data; and the realisation of the manuscript. PP has approved the submitted version (and any substantially modified version that involves the author's contribution to the study) and has agreed both to be personally accountable for the author's own contributions and to ensure that questions related to the accuracy or integrity of any part of the work, even ones in which the author was not personally involved, are appropriately investigated, resolved, and the resolution documented in the literature. ES has made substantial contributions to the acquisition, analysis and interpretation of data and the realisation of the manuscript. ES has approved the submitted version (and any substantially modified version that involves the author's contribution to the study) and has agreed both to be personally accountable for the author's own contributions and to ensure that questions related to the accuracy or integrity of any part of the work, even ones in which the author was not personally involved, are appropriately investigated, resolved, and the resolution documented in the literature. PP has made substantial contributions to the intellectual revision of the work. PP has approved the submitted version (and any substantially modified version that involves the author's contribution to the study) and has agreed both to be personally accountable for the author's own contributions and to ensure that questions related to the accuracy or integrity of any part of the work, even ones in which the author was not personally involved, are appropriately investigated, resolved, and the resolution documented in the literature. AL has made substantial contributions to the design and the intellectual revision of the work. AL has approved the submitted version (and any substantially modified version that involves the author's contribution to the study) and has agreed both to be personally accountable for the author's own contributions and to ensure that questions related to the accuracy or integrity of any part of the work, even ones in which the author was not personally involved, are appropriately investigated, resolved, and the resolution documented in the literature. All authors read and approved the final manuscript.

\section{Funding}

The authors state that this work has not received any funding.

\section{Availability of data and materials}

Not applicable.

\section{Ethics approval and consent to participate}

Institutional Review Board approval was not required because this is an evidence summary and expert recommendation paper. No new studies on human patients were undertaken specifically for this paper. Not applicable.

\section{Consent for publication}

Not applicable.

\section{Competing interests}

The authors of this manuscript declare no competing interests.

\section{Author details}

${ }^{1}$ Department of Surgical and Medical Sciences and Translational Medicine, Sapienza University of Rome - Sant'Andrea University Hospital, Via di Grottarossa, 1035-1039, 00189 Rome, Italy. ${ }^{2}$ Department of Radiology, San Giovanni Addolorata Hospital, Via Dell'Amba Aradam 9, 00184 Rome, Italy.

\section{Received: 25 September 2020 Accepted: 12 January 2021}

\section{Published online: 11 March 2021}

\section{References}

1. Huang C, Wang Y, Li X et al (2020) Clinical features of patients infected with 2019 novel coronavirus in Wuhan, China. Lancet 395:497-506

2. Li Q, Guan X, Wu P et al (2020) Early transmission dynamics in Wuhan, China, of novel coronavirus-infected pneumonia. N Engl J Med 382:1199-1207

3. WHO Director-General's opening remarks at the media briefing on COVID-19-11 March 2020. https://www.who.int/dg/speeches/detail/ who-director-general-s-opening-remarks-at-the-media-briefing-on-covid -19---11-march-2020. Accessed 4 May 2020

4. Hani C, Trieu NH, Saab I et al (2020) COVID-19 pneumonia: a review of typical CT findings and differential diagnosis. Diagn Interv Imaging. https $/ /$ doi.org/10.1016/j.diii.2020.03.014

5. Dai W-C, Zhang H-W, Yu J et al (2020) CT imaging and differential diagnosis of COVID-19. Can Assoc Radiol J 71:195-200

6. Bordi L, Nicastri E, Scorzolini L et al (2020) Differential diagnosis of illness in patients under investigation for the novel coronavirus (SARS-CoV-2), Italy, February 2020. Euro Surveill. https://doi.org/10.2807/1560-7917. ES.2020.25.8.2000170

7. Chan JF-W, Yuan S, Kok K-H et al (2020) A familial cluster of pneumonia associated with the 2019 novel coronavirus indicating person-to-person transmission: a study of a family cluster. Lancet 395:514-523

8. Zhou P, Yang X-L, Wang X-G et al (2020) A pneumonia outbreak associated with a new coronavirus of probable bat origin. Nature 579:270-273

9. Rubin GD, Ryerson CJ, Haramati LB et al (2020) The role of chest imaging in patient management during the COVID-19 pandemic: a multinational consensus statement from the Fleischner society. Radiology 296:172-180

10. Wong HYF, Lam HYS, Fong AH-T et al (2020) Frequency and distribution of chest radiographic findings in patients positive for COVID-19. Radiology 296:E72-E78

11. Jacobi A, Chung M, Bernheim A, Eber C (2020) Portable chest X-ray in coronavirus disease-19 (COVID-19): a pictorial review. Clin Imaging 64:35-42

12. Pan F, Ye T, Sun P et al (2020) Time course of lung changes on chest $C T$ during recovery from 2019 novel coronavirus (COVID-19) pneumonia. Radiology 200370

13. Hansell DM, Bankier AA, MacMahon H et al (2008) Fleischner Society: glossary of terms for thoracic imaging. Radiology 246:697-722

14. Bernheim A, Mei X, Huang M et al (2020) Chest CT findings in coronavirus disease-19 (COVID-19): relationship to duration of infection. Radiology. https://doi.org/10.1148/radiol.2020200463

15. Chung M, Bernheim A, Mei X et al (2020) CT imaging features of 2019 novel coronavirus (2019-nCoV). Radiology 295:202-207

16. Kanne JP, Little BP, Chung JH et al (2020) Essentials for radiologists on COVID-19: an update—radiology scientific expert panel. Radiology. https ://doi.org/10.1148/radiol.2020200527

17. Ciceri F, Beretta L, Scandroglio AM et al (2020) Microvascular COVID-19 lung vessels obstructive thromboinflammatory syndrome (MicroCLOTS): an atypical acute respiratory distress syndrome working hypothesis. Crit Care Resusc 22(2):95-97

18. Thachil J (2020) The versatile heparin in COVID-19. J Thromb Haemost. https://doi.org/10.1111/jth.14821

19. Tang N, Bai H, Chen X et al (2020) Anticoagulant treatment is associated with decreased mortality in severe coronavirus disease 2019 patients with coagulopathy. J Thromb Haemost. https://doi.org/10.1111/jth.14817

20. Sheard S, Rao P, Devaraj A (2012) Imaging of acute respiratory distress syndrome. Respir Care 57:607-612

21. Franquet $T$ (2018) Imaging of community-acquired pneumonia. J Thorac Imaging 33:282-294

22. Walker CM, Abbott GF, Greene RE et al (2014) Imaging pulmonary infection: classic signs and patterns. AJR Am J Roentgenol 202:479-492

23. Ludvigsson JF (2020) Systematic review of COVID-19 in children shows milder cases and a better prognosis than adults. Acta Paediatr 109:1088-1095

24. Koo HJ, Lim S, Choe J, Choi SH, Sung H, Do KH (2018) Radiographic and CT features of viral pneumonia. Radiographics 38:719-739

25. Fujii T, Nakamura T, I wamoto A (2007) Pneumocystis pneumonia in patients with HIV infection: clinical manifestations, laboratory findings, and radiological features. J Infect Chemother 13:1-7

26. Kuhlman JE, Kavuru M, Fishman EK, Siegelman SS (1990) Pneumocystis carinii pneumonia: spectrum of parenchymal CT findings. Radiology 175:711-714

27. Franquet T, Müller NL, Giménez A et al (2001) Spectrum of pulmonary aspergillosis: histologic, clinical, and radiologic findings. Radiographics 21:825-837

28. Denning DW (1998) Invasive aspergillosis. Clin Infect Dis 26:781-803

29. Davda S-Y, Kowa X, Aziz Z et al (2018) The development of pulmonary aspergillosis and its histologic, clinical, and radiologic manifestations. ClinRadiol 73:913-921 
30. Gluecker T, Capasso P, Schnyder P et al (1999) Clinical and radiologic features of pulmonary edema. Radiographics 19:1507-1531

31. Chen CC, Zhou Y, Wang DW (2020) SARS-CoV-2: a potential novel etiology of fulminant myocarditis. Herz 45:230-232

32. Han D, Lee KS, Franquet T et al (2003) Thrombotic and nonthrombotic pulmonary arterial embolism: spectrum of imaging findings. Radiographics 23:1521-1539

33. Marongiu F, Grandone E, Barcellona D (2020) Pulmonary thrombosis in 2019-nCoV pneumonia? J Thromb Haemost. https://doi.org/10.1111/ jth.14818

34. Porfidia A, Valeriani E, Pola R et al (2020) Venous thromboembolism in patients with COVID-19: Systematic review and meta-analysis. Thrombos Res 196:67-74. https://doi.org/10.1016/j.thromres.2020.08.020

35. Langford CA (2003) Treatment of ANCA-associated vasculitis. N Engl J Med 349:3-4

36. Adams TN, Zhang D, Batra K, Fitzgerald JE (2018) Pulmonary manifestations of large, medium, and variable vessel vasculitis. Respir Med 145:182-191

37. Castañer E, Alguersuari A, Andreu M et al (2012) Imaging findings in pulmonary vasculitis. Semin Ultrasound CT MR 33:567-579

38. Ando Y, Okada F, Matsumoto S, Mori H (2004) Thoracic manifestation of myeloperoxidase-antineutrophil cytoplasmic antibody (MPO-ANCA)related disease. CT findings in 51 patients. J Comput Assist Tomogr 28:710-716

39. Cox CW, Rose CS, Lynch DA (2014) State of the art: imaging of occupational lung disease. Radiology 270:681-696

40. Cormier Y, Brown M, Worthy S et al (2000) High-resolution computed tomographic characteristics in acute farmer's lung and in its follow-up. Eur Respir J 16:56-60

41. Hirschmann JV, Pipavath SNJ, Godwin JD (2009) Hypersensitivity pneumonitis: a historical, clinical, and radiologic review. Radiographics 29:1921-1938

42. Franquet T, Hansell DM, Senbanjo T et al (2003) Lung cysts in subacute hypersensitivity pneumonitis. J Comput Assist Tomogr 27:475-478

43. Silva CIS, Churg A, Müller NL (2007) Hypersensitivity pneumonitis: spectrum of high-resolution CT and pathologic findings. AJR Am J Roentgenol 188:334-344

44. Webb WR, Müller NL, Naidich DP (1996) High-resolution CT of the lung. Lippincott Williams \& Wilkins, Philadelphia

45. Silva CIS, Müller NL, Lynch DA et al (2008) Chronic hypersensitivity pneumonitis: differentiation from idiopathic pulmonary fibrosis and nonspecific interstitial pneumonia by using thin-section CT. Radiology 246:288-297

46. Jeong YJ, Kim K-I, Seo IJ et al (2007) Eosinophilic lung diseases: a clinical, radiologic, and pathologic overview. Radiographics 27:617-637 ((discussion 637-9))

47. Allen JN, Davis WB (1994) Eosinophilic lung diseases. Am J Respir Crit Care Med 150:1423-1438

48. Johkoh T, Müller NL, Akira M et al (2000) Eosinophilic lung diseases: diagnostic accuracy of thin-section CT in 111 patients. Radiology 216:773-780

49. King MA, Pope-Harman AL, Allen JN et al (1997) Acute eosinophilic pneumonia: radiologic and clinical features. Radiology 203:715-719

50. Daimon T, Johkoh T, Sumikawa H et al (2008) Acute eosinophilic pneumonia: thin-section CT findings in 29 patients. Eur J Radiol 65:462-467
51. Ebara H, Ikezoe J, Johkoh T et al (1994) Chronic eosinophilic pneumonia: evolution of chest radiograms and CT features. J Comput Assist Tomogr 18:737-744

52. Jederlinic PJ, Sicilian L, Gaensler EA (1988) Chronic eosinophilic pneumonia a report of 19 cases and a review of the literature. Medicine (Baltimore) 67:154-162

53. Marom EM, McAdams HP, Erasmus JJ, Goodman PC (1999) The many faces of pulmonary aspiration. AJR Am J Roentgenol 172:121-128

54. Kim M, Lee KY, Lee KW, Bae KT (2008) MDCT evaluation of foreign bodies and liquid aspiration pneumonia in adults. AJR Am J Roentgenol 190:907-915

55. Betancourt SL, Martinez-Jimenez S, Rossi SE et al (2010) Lipoid pneumonia: spectrum of clinical and radiologic manifestations. AJR Am J Roentgenol 194:103-109

56. Baron SE, Haramati LB, Rivera VT (2003) Radiological and clinical findings in acute and chronic exogenous lipoid pneumonia. J Thorac Imaging 18:217-224

57. Lee KS, Müller NL, Hale V et al (1995) Lipoid pneumonia. J Comput Assist Tomogr 19:48-51

58. Brechot JM, Buy JN, Laaban JP, Rochemaure J (1991) Computed tomography and magnetic resonance findings in lipoid pneumonia. Thorax 46:738-739

59. Seymour JF, Presneill JJ (2002) Pulmonary alveolar proteinosis: progress in the first 44 years. Am J Respir Crit Care Med 166:215-235

60. Trapnell BC, Whitsett JA, Nakata K (2003) Pulmonary alveolar proteinosis. N Engl J Med 349:2527-2539

61. Trapnell BC, Nakata K, Bonella F et al (2019) Pulmonary alveolar proteinosis. Nat Rev Dis Primers 5:16

62. Rossi SE, Erasmus JJ, McAdams HP et al (2000) Pulmonary drug toxicity: radiologic and pathologic manifestations. Radiographics 20:1245-1259

63. Ellis SJ, Cleverley JR, Müller NL (2000) Drug-induced lung disease: highresolution CT findings. AJR Am J Roentgenol 175:1019-1024

64. Kuhlman JE, Teigen C, Ren $\mathrm{H}$ et al (1990) Amiodarone pulmonary toxicity: CT findings in symptomatic patients. Radiology 177:121-125

65. Rodríguez-Moreno A, Ridao N, García-Ledesma P et al (2009) Sirolimus and everolimus induced pneumonitis in adult renal allograft recipients: experience in a center. Transpl Proc 41:2163-2165

66. Ikeda M, Tanaka H, Sadamatsu K (2011) Diffuse alveolar hemorrhage as a complication of dual antiplatelet therapy for acute coronary syndrome. Cardiovasc Revasc Med 12:407-411

67. Sterrett C, Brownfield J, Korn CS et al (2003) Patterns of presentation in heroin overdose resulting in pulmonary edema. Am J Emerg Med 21:32-34

68. Mabry B, Greller HA, Nelson LS (2004) Patterns of heroin overdoseinduced pulmonary edema. Am J Emerg Med 22:316

69. Restrepo CS, Carrillo JA, Martínez S et al (2007) Pulmonary complications from cocaine and cocaine-based substances: imaging manifestations. Radiographics 27:941-956

\section{Publisher's Note}

Springer Nature remains neutral with regard to jurisdictional claims in published maps and institutional affiliations. 